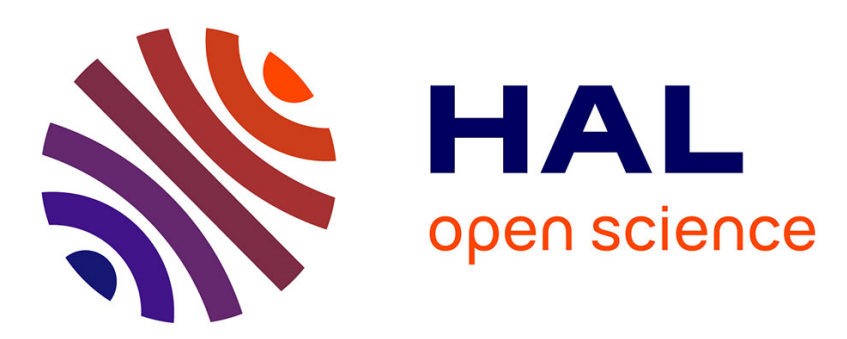

\title{
Biophysical mechanisms underlying the paradoxical potentiation of the low-voltage activated calcium current in thalamocortical neurons: a modeling study
}

Régis C Lambert, Thomas Bessaïh, Nathalie Leresche

\section{- To cite this version:}

Régis C Lambert, Thomas Bessaïh, Nathalie Leresche. Biophysical mechanisms underlying the paradoxical potentiation of the low-voltage activated calcium current in thalamocortical neurons: a modeling study. Thalamus and Related Systems, 2005, 3 (3), pp.165-173. 10.1017/S1472928807000179 . hal-01370870

\author{
HAL Id: hal-01370870 \\ https://hal.science/hal-01370870
}

Submitted on 23 Sep 2016

HAL is a multi-disciplinary open access archive for the deposit and dissemination of scientific research documents, whether they are published or not. The documents may come from teaching and research institutions in France or abroad, or from public or private research centers.
L'archive ouverte pluridisciplinaire HAL, est destinée au dépôt et à la diffusion de documents scientifiques de niveau recherche, publiés ou non, émanant des établissements d'enseignement et de recherche français ou étrangers, des laboratoires publics ou privés. 


\title{
Biophysical mechanisms underlying the paradoxical potentiation of the low-voltage activated calcium current in thalamocortical neurons: a modeling study
}

\author{
REGIS C. LAMBERT, THOMAS BESSA ̈̈H AND NATHALIE LERESCHE \\ Université Pierre et Marie Curie-Paris6, UMR 7102-CNRS, Paris, France
}

\begin{abstract}
In thalamocortical neurons relaying sensory information, we recently described a phosphorylation mechanism that induces a marked increase in the amplitude of the low-voltage activated $\mathrm{Ca}^{2+}$ current (T-type). Surprisingly, potentiation of the T-current closely depends on the state of the channel and is, therefore, both voltage- and ATP-dependent. Further analysis of the modification of channel activity induced by this regulation, and underlying the increase in the macroscopic current amplitude, requires a detailed study of the T-current biophysical properties that, unfortunately, might be constrained by the technical limitations of whole-cell recordings. Therefore, in the present study we have developed an alternative approach that is based on computational models of T-channel activity using Markov gating schemes. We show that both modifications in the activation kinetics of the channels and/or the existence of a second channel population with a conducting state conditioned by a phosphorylation step can explain the specific properties of T-currents that have been observed in thalamocortical neurons as a result of their ATP/voltage-dependent regulation. The flexibility in the T-type current behavior that is incorporated in these models might also help to unravel new roles for T-channels in shaping the different firing properties of thalamocortical neurons.
\end{abstract}

Keywords: Markov kinetic scheme, T-type, regulation, channel phosphorylation

\section{INTRDDUCTION}

Following the pioneering work of the groups of Llinas and Steriade (Llinas and Jahnsen, 1982; Deschenes et al., 1984; Jahnsen and Llinas, 1984), many studies have furthered our understanding of low-threshold $\mathrm{Ca}^{2+}$ potentials/ currents and their contribution to shaping the firing output of thalamic neurons (Crunelli et al., 1987; Coulter et al., 1989; Crunelli et al., 1989; Hernandez-Cruz and Pape, 1989; Huguenard and Prince, 1992; Zhan et al., 1999). The emphasis has been on the role of these $\mathrm{Ca}^{2+}$ potentials both in normal brain functions, such as various slow oscillatory activities that occur during different stages of sleep (Steriade et al., 1993), and in several major human disorders such as absence seizures and thalamocortical disrhythmias (which has been suggested to include neurogenic pain, depression, Parkinson and tinnitus) (Llinas et al., 1999).

By contrast, few studies have analyzed the regulation of low threshold $\mathrm{Ca}^{2+}$ currents (T-type $\mathrm{Ca}^{2+}$ currents) in thalamocortical neurons that express mainly $\mathrm{T}$-channels of the Cav3.1 type (Reviewed in Lambert et al., 2006). Recently, we have described a phosphorylation-mediated potentiation of the T-current that might modify our classical view of the T-channel contribution to thalamocortical excitability (Leresche et al., 2004). This channel phosphorylation leads to a 1.5 -fold increase in current amplitude, and only

Corresponding author:

R.C. Lambert

Email: regis.lambert@snv.jussieu.fr occurs when the T-type channels remain inactivated for a few hundreds of milliseconds (time constant $\sim 300 \mathrm{msec}$ ). Conversely, the slow dephosphorylation process takes place at relatively hyperpolarized potentials where channels remain in the closed states (time constant $\sim 3 \mathrm{sec}$ ). As a consequence, ATP-dependent regulation presents a novel voltage dependence that follows closely the steady-state voltagedependence of the current. In addition, this regulation is only found in native neurons where T-channels have a predominant dendritic location (Destexhe et al., 1998; Williams and Stuart, 2000), and is not present in either expression systems or in nucleated patches excised from thalamocortical neurons. Therefore, a detailed analysis of the phosphorylation-induced changes in the biophysical properties of the T-type channels is constraint by the limited voltage-control that can be achieved under whole-cell clamp in situ. An alternative to this is to use a modelling approach that allows testing of various hypotheses concerning the potential mechanisms that might contribute to the increased T-current amplitude.

Several groups have successfully developed models of the T-current and shown, in particular, how its interaction with other conductances leads to the various oscillatory activities that are characteristic of thalamocortical neurons (Wang et al., 1991; Huguenard and McCormick, 1992; McCormick and Huguenard, 1992; Toth and Crunelli, 1992; Williams et al., 1997; Destexhe et al., 1998). However, since our study requires changing the transition between the different states of channel activity, here we develop a new model based on a multistate Markovian scheme. We show that the potentiation of the macroscopic T-current can be explained by changes in the activation 
kinetics of the channel and/or the existence of a specific population of channels that conducts $\mathrm{Ca}^{2+}$ only when phosphorylated.

\section{METHODS}

\section{In vitro recordings}

\section{SLICES}

The brain was excised from 8-12-day-old Wistar rats. A block of tissue containing the thalamus was removed and placed in a cold $\left(<4{ }_{4}^{\circ} \mathrm{C}\right)$ oxygenated $\left(95 \% \mathrm{O}_{2}: 5 \% \mathrm{CO}_{2}\right)$ solution of artificial cerebrospinal fluid (in $\mathrm{mM}$ ): $125 \mathrm{NaCl}, 2.5 \mathrm{KCl}, 2$ $\mathrm{CaCl}_{2}, 1 \mathrm{MgCl}_{2}, 1.25 \mathrm{NaH}_{2} \mathrm{PO}_{4}, 26 \mathrm{NaHCO}_{3}$ and 25 glucose. The block of tissue was glued, ventral-surface uppermost, to the stage of a vibroslice (Leica VT100oS), and $220 \mu \mathrm{m}$-thick horizontal sections containing the ventrobasal nucleus and the nucleus reticularis were prepared by using the internal capsule and the medial lemniscus as landmarks. Slices were kept at $32^{\circ} \mathrm{C}$ in the oxygenated solution for at least 1 hour before being transferred to the experimental setup, where they were perfused at a rate of $1-2 \mathrm{ml} \mathrm{min}^{-1}$ at $25^{\circ} \mathrm{C}$.

\section{WHOLE-CELL RECORDINGS}

Membrane currents were recorded in the whole-cell configuration of the patch clamp technique using an Axopatch $200 \mathrm{~B}$ amplifier (Axon Instruments). Thalamic neurons were visualized under Nomarski optics (Zeiss Axioskop, $\times 60$ lens). Recording pipettes were pulled from borosilicate glass (WPI TW15oF) and coated with wax. Electrodes were filled with the following solution (in $\mathrm{mM}$ ): $110 \mathrm{CsCl}, 1 \mathrm{CaCl}_{2}, 5 \mathrm{MgCl}_{2}$, 10 Bapta, 10 HEPES, and 4 Na- ATP, 15 phosphocreatine, 50 units $\mathrm{ml}^{-1}$ creatine phosphokinase, $\mathrm{pH} 7.3$, osmolarity 305 mOsm.

Values of access resistance ranged from $4-6 \mathrm{M} \Omega$ at the beginning of the recording to $4-10 \mathrm{M} \Omega$ at the end. At least $70 \%$ of the cell capacitance and series resistances were compensated. Voltage protocols and acquisition were controlled by Axograph 4.8, and the holding potential set to $-60 \mathrm{mV}$. Membrane currents were filtered by a 4-pole Bessel filter set at a corner frequency of $5 \mathrm{kHz}$, digitalized on line at $10 \mathrm{kHz}$ and analyzed later using IgorPro 4.0 home-made routines. Leak currents were subtracted off-line using templates constructed from the current induced by a $10 \mathrm{mV}$ hyperpolarizing step applied from $-100 \mathrm{mV}$ at the end of each episode. To isolate $\mathrm{Ca}^{2+}$ currents and suppress any GABAergic synaptic currents the following were added to the perfusion medium, $10 \mathrm{mM}$ TEA, $1 \mathrm{mM}$ 4-aminopyridine, $0.5 \mu \mathrm{M}$ tetrodotoxin (Tocris), $2 \mathrm{mM} \mathrm{CsCl}$ and $1 \mu \mathrm{M}$ SR95531 (Tocris).

\section{Modeling methods}

The simulations were performed under the Neuron 5.8 environment (Hines and Carnevale, 1997), and the multistate Markov model designed to describe the activity of the T-type channels in thalamocortical neurons was derived from the model proposed by Burgess et al. (Burgess et al., 2002) for Cav3.1 channels. Rate constants were adjusted by trial and error to simulate macroscopic T-currents displaying typical biophysical properties used in previous modeling studies (Wang et al., 1991; Huguenard and McCormick, 1992; McCormick and Huguenard, 1992; Toth and Crunelli, 1992;

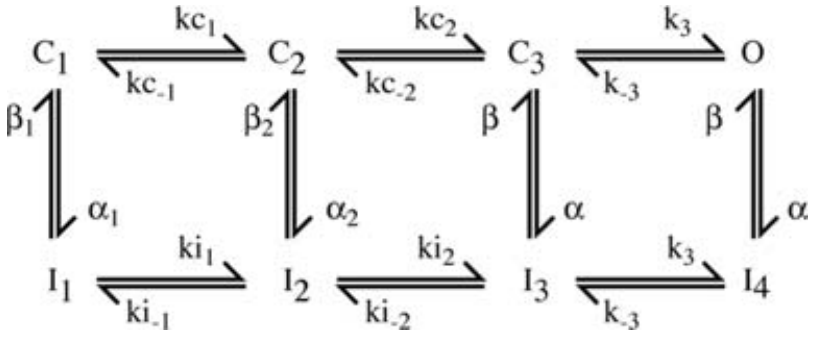

Fig. 1. Kinetic scheme for gating of low-voltage activated $\mathrm{Ca}^{2+}$ channels. All rate constants except $\mathrm{k}_{3}$ on the top and bottom rows of the model are voltage-dependent. The rate constants for inactivation and recovery from inactivation are voltage-independent.

Williams et al., 1997; Destexhe et al., 1998). Parameters were constrained further by invoking microscopic reversibility.

As illustrated in Fig. 1, the kinetic scheme comprised three closed $\left(\mathrm{C}_{1}, \mathrm{C}_{2}\right.$ and $\left.\mathrm{C}_{3}\right)$ and one open $(\mathrm{O})$ state, each interconnected with an inactivated state ( $\mathrm{I}_{1}, \mathrm{I}_{2}, \mathrm{I}_{3}$ and $\left.\mathrm{I}_{4}\right)$, and is, therefore, composed of three loops. Following Burgess et al. (Burgess et al., 2002), the transitions between closed states and between inactivated states are voltage-dependent, but transitions between closed and inactivated states are voltage-independent, as is the open-to-inactivated state transition. This voltage-independence is in agreement with the lack of voltage-dependence of the inactivation process described for native (Chen and Hess, 1990) and reconstituted channels (Serrano et al., 1999). Rate constants for the transition $\mathrm{C}_{3}$ to $\mathrm{O}\left(\mathrm{C}_{3}\right.$ to $\left.\mathrm{I}_{3}\right)$ were equalized to those for transition $\mathrm{I}_{3}$ to $\mathrm{I}_{4}$ ( $\mathrm{O}$ to $\mathrm{I}_{4}$, respectively). The voltage-dependent rate constants were described by the following equation:

In the activation (forward) direction

$$
\mathrm{kx}_{\mathrm{n}}(\mathrm{V})=\mathrm{kx}_{\mathrm{n}} \exp \left[\mathrm{d}_{\mathrm{n}} \mathrm{q}_{\mathrm{n}} \mathrm{V} / \mathrm{T}\right]
$$

In the deactivation (backward) direction

$$
\mathrm{kx}_{-\mathrm{n}}(\mathrm{V})=\mathrm{kx}_{-\mathrm{n}} \exp \left[\left(1-\mathrm{d}_{\mathrm{n}}\right) \mathrm{q}_{\mathrm{n}} \mathrm{V} / \mathrm{T}\right]
$$

where $\mathrm{x}=\mathrm{c}$ for transition between closed states or i for transition between inactivated states; $\mathrm{n}=1,2$ or 3 indicating the corresponding loop in the kinetic scheme (numbered from left to right); $\mathrm{V}$ is the membrane potential (in $\mathrm{mV}$ ); $\mathrm{T}=25.6 \mathrm{mV}$ represents the thermal energy associated with the experimental temperature $\left(25^{\circ} \mathrm{C}\right)$; $\mathrm{q}_{\mathrm{n}}$ represents the gating charge associated with the $n_{\text {th }}$ loop in the model; and $d_{n}$ represents the fraction of the voltage-dependence associated with kinetics of the transition in the forward direction.

Following Burgess et al. (Burgess et al., 2002), d 3 was set to o and, therefore, in the third loop, only backward transitions from $\mathrm{O}$ to $\mathrm{C}_{3}$ and from $\mathrm{I}_{4}$ to $\mathrm{I}_{3}$ are voltage-dependent, which reflects the voltage dependence of the tail current kinetics that was observed for large hyperpolarizations (Serrano et al., 1999). Parameter values used to model voltage-dependent and -independent transitions are summarized in Table 1 and Table 2 , respectively.

\section{RESULTS}

The increase in current amplitude generated by the phosphorylated channels is not associated with changes in the 
Table 1. Voltage-dependent rate constants.

\begin{tabular}{|c|c|c|c|c|c|c|c|c|c|c|c|c|}
\hline \multirow[t]{3}{*}{ Mode 1} & $\mathrm{Kc}_{1}$ & 9 & $\mathrm{Kc}_{-1}$ & 0.162 & $\mathrm{Ki}_{1}$ & 0.86 & $\mathrm{Ki}_{-1}$ & $6 \times 10^{-3}$ & $\mathrm{~d}_{1}$ & 0.8 & $\mathrm{q}_{1}$ & 2.24 \\
\hline & $\mathrm{Kc}_{2}$ & 13.05 & $\mathrm{Kc}_{-2}$ & $7.47 \times 10^{-3}$ & $\mathrm{Ki}_{2}$ & 0.71 & $\mathrm{Ki}_{-2}$ & $4.3 \times 10^{-7}$ & $\mathrm{~d}_{2}$ & 0.26 & $\mathrm{q}_{2}$ & 3.81 \\
\hline & $\mathrm{Kc}_{3}$ & 0.16 & $\mathrm{Kc}_{-3}$ & $3.15 \times 10^{-3}$ & & & & & $\mathrm{~d}_{3}$ & o & $\mathrm{q}_{3}$ & 0.79 \\
\hline \multirow[t]{3}{*}{ Mode 2} & $\mathrm{~K}^{\prime} \mathrm{c}_{1}$ & 40 & $\mathrm{~K}^{\prime} \mathrm{c}_{-1}$ & 0.72 & & & & & & & & \\
\hline & $\mathrm{K}^{\prime} \mathrm{c}_{2}$ & 58 & $\mathrm{~K}^{\prime} \mathrm{c}_{-2}$ & $3.32 \times 10^{-2}$ & & & & & & & & \\
\hline & $\mathrm{K}^{\prime} \mathrm{c}_{3}$ & 0.88 & $\mathrm{~K}^{\prime} \mathrm{c}_{-3}$ & $1.4 \times 10^{-2}$ & & & & & & & & \\
\hline
\end{tabular}

Rate constant $\mathrm{kx}_{\mathrm{n}}$ and $\mathrm{k}^{\prime} \mathrm{x}_{\mathrm{n}}$ in $\mathrm{ms}{ }^{-1}$. The values $\mathrm{k}^{\prime} \mathrm{x}_{\mathrm{n}}$ were used in Fig. $2 \mathrm{~A}, \mathrm{~B}$ to simulate currents with faster activation kinetics (scheme in Fig. 1 ) and in Fig. 3 for the activation transition of the mode 2 (scheme in Fig. $2 \mathrm{C}$ ).

voltage-dependence of both current activation and inactivation. At the microscopic level, therefore, two main hypotheses can be made to explain the increase in current amplitude.

Because the amplitude of the macroscopic current results from the interplay between the channel activation and inactivation processes, an acceleration in the kinetics of the channel activation can potentially generate a marked increase in current amplitude. Indeed, some of our experimental data support this hypothesis. Although the space-clamp quality in thalamocortical neurons and the filtering properties of their dendritic arborization preclude a detailed analysis of the fast rise time of the T-currents, a clear acceleration in the rise time of the potentiated current was observed in some cases (Fig. 2A1). To test this hypothesis further we compared the amplitudes of currents generated by the model described in Fig. 1 when different rate constant values are used in the activation pathway (C-C and C-O transitions; see Table 1 for $\mathrm{kc}_{\mathrm{n}}$ and $\mathrm{k}^{\prime} \mathrm{c}_{\mathrm{n}}$ values). As illustrated in Fig. 2A2, a marked increase in current amplitude was observed with faster activation kinetics. The voltage dependence of channel activation was affected only slightly (voltage for half-activation estimated by the fit of Boltzmann equations to the IV curves: $-50.5 \mathrm{mV}$ and $-54.5 \mathrm{mV}$ for the model with slow and fast kinetics, respectively) and the voltage-sensitivity of the steady-state inactivation was unaffected (voltage for half-inactivation, $-81.8 \mathrm{mV}$ and $-82.5 \mathrm{mV}$ for the model with slow and fast kinetics respectively). As might be expected, even in the absence of any modification in the voltage-independent transition toward inactivated states (C-I and O-I transitions), the acceleration in activation kinetics increases the apparent inactivation kinetics of the macroscopic current (Fig. 2B). This property of the simulated current agrees with our experimental recordings showing that phosphorylated channels generate macroscopic currents with faster inactivation kinetics than non-phosphorylated ones (acceleration in the inactivation time constant $\sim 1.5 \mathrm{msec}$ ) (Fig. 2A1). The major features of the T-current potentiation observed in thalamocortical neurons, therefore, can be modeled by a multistate Markov scheme in which the channels operate in two modes that differ only in their activation kinetics (Fig. 2C). Because channel phosphorylation occurs exclusively when the channels remain inactivated for a few hundred milliseconds, the non-phosphorylated mode

Table 2. Voltage-independent rate constants (in $\mathrm{msec}^{-1}$ ).

\begin{tabular}{lrlll}
\hline Mode 1 & $\alpha_{1}$ & $8.0 \times 10^{-4}$ & $\beta_{1}$ & $3.0 \times 10^{-3}$ \\
& $\alpha_{2}$ & $2.3 \times 10^{-3}$ & $\beta_{2}$ & $3.3 \times 10^{-3}$ \\
& $\alpha$ & $6.5 \times 10^{-2}$ & $\beta$ & $1.0 \times 10^{-4}$ \\
Mode 2 & $\alpha^{\prime}$ & $7.0 \times 10^{-2}$ & $\beta^{\prime}$ & $1.08 \times 10^{-4}$
\end{tabular}

The values of $\alpha^{\prime}$ and $\beta^{\prime}$ were used for the inactivation transitions $\mathrm{C}_{3}^{\prime} \mathrm{I}_{3}^{\prime}$ and $\mathrm{O}^{\prime}-\mathrm{I}_{4}^{\prime}$ of mode 2 in the scheme of Fig. $2 \mathrm{C}$. (mode 1, characterized by the slowest activation kinetics) and the phosphorylated mode (mode 2, associated with the fastest kinetics) are connected by voltage-independent transitions between their inactivated states. The rate constants of these In-I'n transitions greatly favor the forward direction (mode 1 to mode 2), and we hypothesized that transition occurring at hyperpolarized potentials are unlikely (Table 3 ). The backward transition between mode 2 and mode 1 , which corresponds to the dephosphorylation process, takes place slowly when the channel remains in closed states. The two modes, therefore, are also connected by voltage-independent transitions between their closed states, with the rate constants favoring the transition from C'n (mode 2 ) to Cn (mode 1) (Table 3 ). Using this model, we were able to generate a T-type current that displays the biophysical characteristics (Fig. 3) that we had described previously for native current in thalamocortical neurons (Leresche et al., 2004). The recovery from inactivation of the simulated current has a characteristic biphasic shape due to the potentiation of the current amplitude that occurs with on-time and off-time constants of $336 \mathrm{msec}$ and $2.7 \mathrm{sec}$, respectively (Fig. $3 \mathrm{~A}, \mathrm{C}$ ). This potentiation displays a voltagesensitivity that is similar to the steady-state inactivation (voltage of half potentiation $=-85.2 \mathrm{mV}$ ) (Fig. $3 \mathrm{~B}, \mathrm{D}$ ), and induces minor changes in the activation voltage-sensitivity (Fig. ${ }_{3} \mathrm{E}$ ) but a clear increase in the inactivation kinetics (see current traces in Fig. $3 \mathrm{~B}$ ).

Another hypothesis that might explain the potentiation of the T-current involves the existence of a specific T-channel population that conducts calcium ions only when phosphorylated. To test this hypothesis, simulations were carried out where the global $\mathrm{T}$ current was generated by two channel populations of equal size: the first channel population obeys the simple kinetics scheme of Fig. 1, and the second population follows the more complicated scheme presented in Fig. $2 \mathrm{C}$, which has been modified so that the open state of mode 1 is a non-conducting state. All the rate constants in modes 1 and 2 were similar except that those governing the transitions between open and inactivated states were increased to simulate the accelerated inactivation kinetics of the potentiated current (see Mode 2, Table 2). As illustrated in Fig. 4, the macroscopic T-current generated by these two channel populations also displays the major characteristics of the T-current behavior that had previously been observed in thalamocortical neurons (Leresche et al., 2004). However, our simulation generates a complex steady-state inactivation curve with an unexpected increase in current amplitude with conditioning prepulses between $-100 \mathrm{mV}$ and $-80 \mathrm{mV}$ (Fig. 4D). The increase in current amplitude can be explained by the ionic flux through the newly phosphorylated/recruited channels that counteracts the expected decrease in current amplitude due to the moderate channel inactivation that occurs at these potentials. 
A1.

A2.
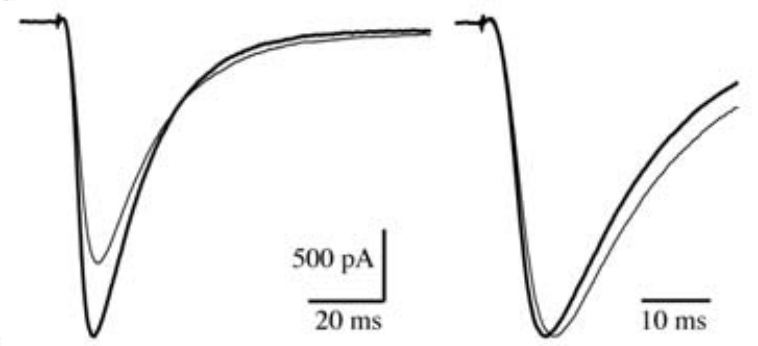
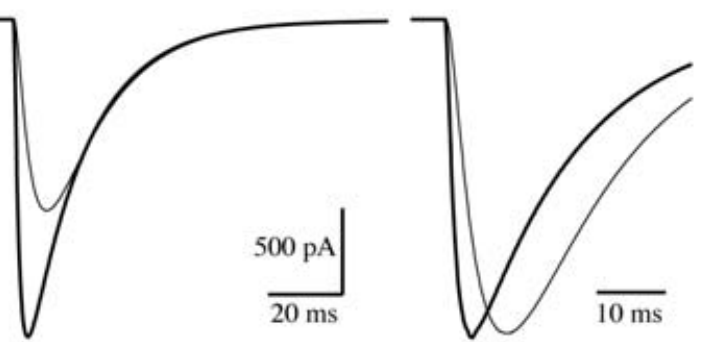

B.

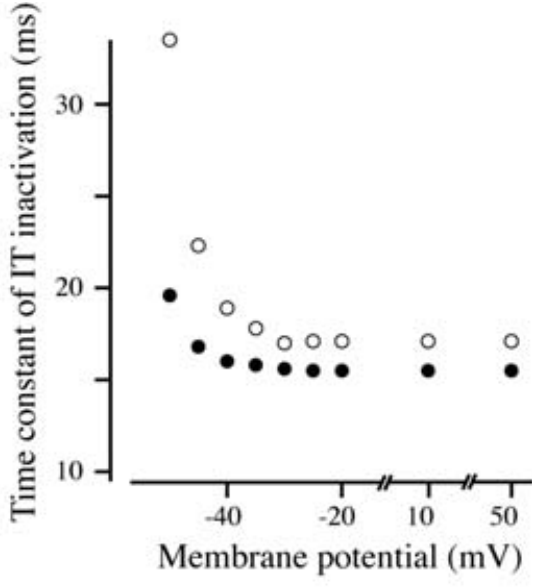

C. Mode 1

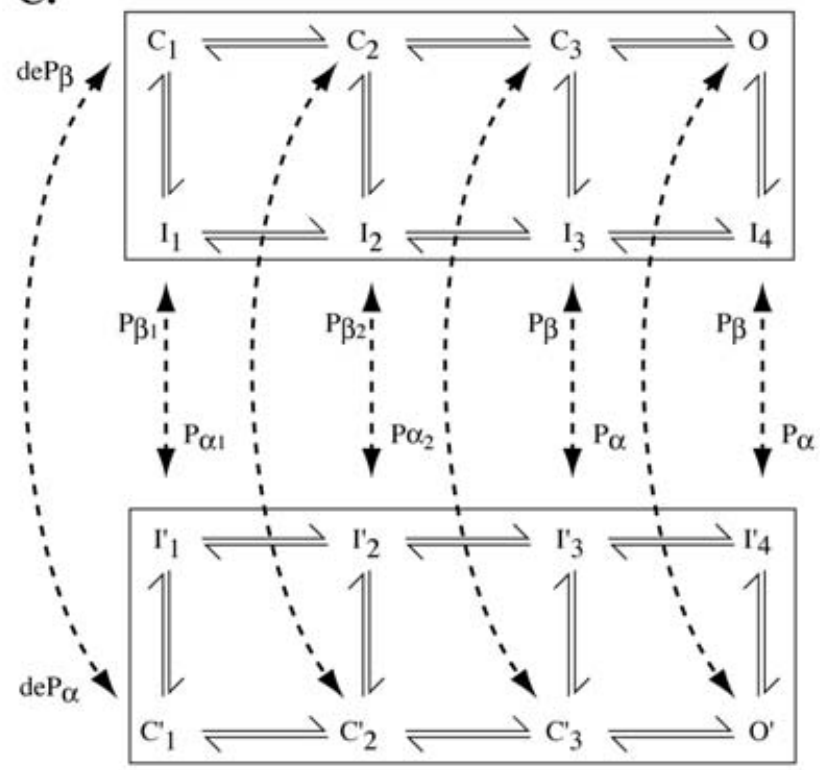

Mode 2

Fig. 2. Acceleration of the activation kinetics of the channel increases both the current amplitude and its inactivation kinetics. A1. The current traces superimposed in $\mathrm{A} 1$ were evoked at $-50 \mathrm{mV}$ in a thalamocortical neuron of the ventrobasal complex after a preceding conditioning prepulse of 10 sec (thin trace) or $1 \mathrm{sec}$ (thick trace) at $-100 \mathrm{mV}$. During the $10 \mathrm{sec}$ hyperpolarizing prepulse, the channels remained closed long enough to be dephosphorylated. Conversely, the $1 \mathrm{sec}$ prepulse allowed full recovery from inactivation with minimal dephosphorylation of the channels. Note the differences in current amplitude and inactivation kinetics. Right panel: traces are normalized to outline the faster rise time of the potentiated current, which was clearly visible in this neuron despite the filtering properties of its dendritic arborization. A2. Simulated currents are shown that were generated by a depolarizing step from $-100 \mathrm{mV}$ to $-30 \mathrm{mV}$ using two sets of values for the rate constant governing the activation of the channel (top row in the scheme in Fig. 1). Currents displaying fast (thick trace) and slow (thin trace) activation kinetics were obtained with the $\mathrm{kcn}$ and $\mathrm{k}^{\prime} \mathrm{cn}$ parameter values presented in Table 1 . Note that an acceleration in the activation kinetics induces a large increase in current amplitude. B. Using the same parameter values, simulated currents were evoked at different potentials, and the time constant of the current inactivation evaluated by exponential fit of the current traces. Note that currents with fast activation kinetics (plain circles) also present faster inactivation kinetics. C. Scheme for the transition between gating modes with different activation kinetics. Transition between mode 1 (which displays slow activation kinetics) and mode 2 (which has fast activation kinetics) occurs upon phosphorylation when channels are inactivated. The backward transition to mode 1 occurs when channels are in closed states.

Table 3. Rate constants of the transition between modes (in $\mathrm{msec}^{-1}$ ).

$\mathrm{P}_{1} 1.0 \times 10^{-8} \mathrm{P} \beta_{1} 1.0 \times 10^{-8} \mathrm{deP} \alpha 1.0 \times 10^{-7} \mathrm{deP} \beta 3.8 \times 10^{-4}$

$\mathrm{P} \alpha_{2} 1.0 \times 10^{-6} \mathrm{P} \beta_{2} 1.0 \times 10^{-6}$

$\mathrm{P} \alpha \quad 3.3 \times 10^{-3} \mathrm{P} \beta \quad 1.0 \times 10^{-6}$
The presence of this phenomenon should depend on the relative voltage sensitivity of the inactivation process of both populations of channel. A more depolarized voltagedependence of the inactivation of the channel population that is sensitive to phosphorylation should indeed shift the recruitment of the newly conducting channels towards more depolarized potentials, and, therefore, either reduce or 
suppress the unexpected rebound in current amplitude of the steady-state inactivation curve.

\section{CONCLUSION}

Our modeling study shows that both modification of the activation kinetics of the T-type $\mathrm{Ca}^{2+}$ channels and/or the existence of a second T-type channel population with a conducting state conditioned by a phosphorylation step can produce macroscopic currents that display the characteristic properties of potentiated and non-potentiated T-currents that have previously been observed in situ. Thus both mechanisms might contribute to the ATP- and voltage-dependent regulation of the low-voltage-activated $\mathrm{Ca}^{2+}$ channels in thalamocortical neurons, with the two mechanisms not mutually exclusive.

\section{DISCUSSIDN}

As suggested by our modeling study, the potentiation of the T-current can be explained by an acceleration in the activation kinetics of the channels. However, the expected faster rise time for the potentiated current than the non-potentiated current was observed in only a small population of the

A
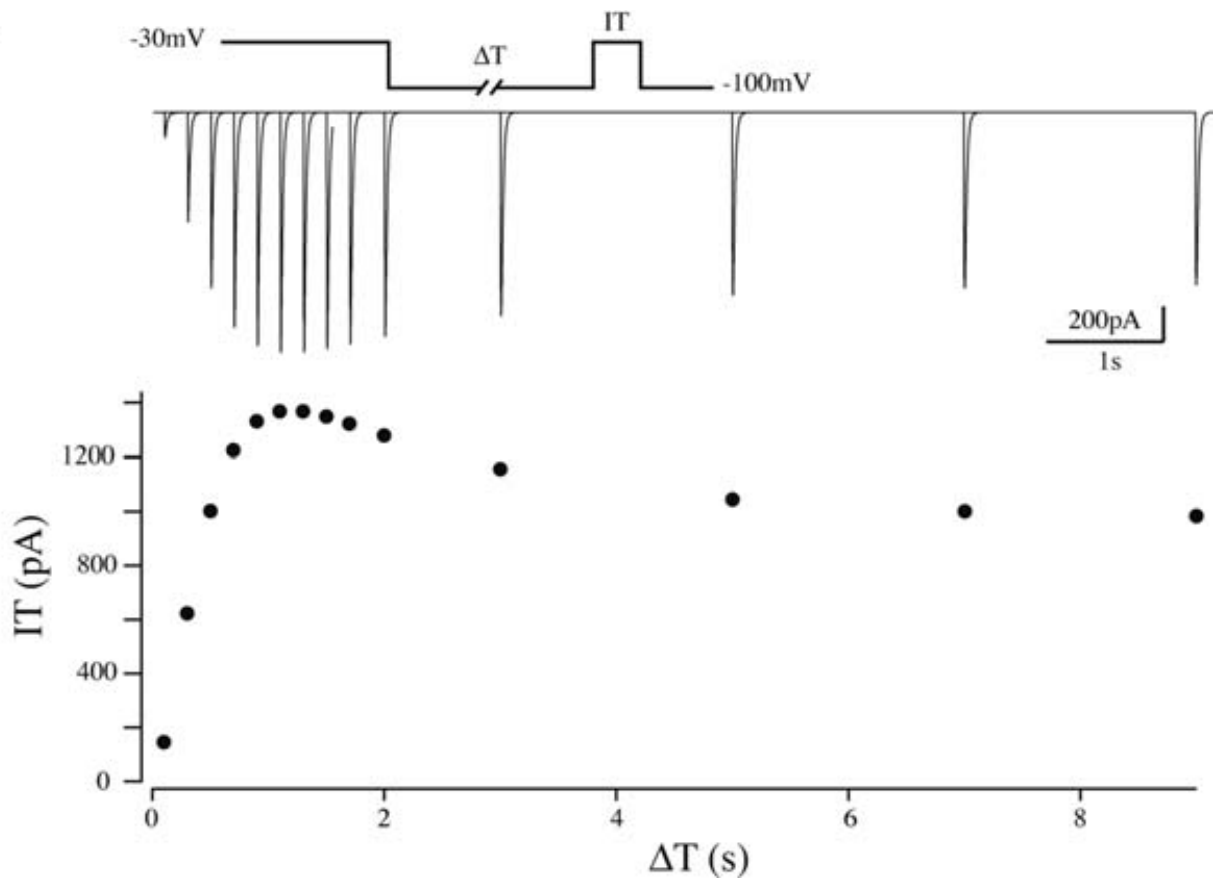

Fig. 3. Phosphorylation-dependent acceleration of the activation kinetics of the channels mimics the major features of the T-current potentiation. The simulation used to construct these graphs were obtained with the kinetics Markov scheme presented in Fig. 2C. The data presented here should be compared with the corresponding figures obtained with native T-current (Leresche et al., 2004). A. Recovery from inactivation of the simulated current. The graph shows the amplitude of the simulated $\mathrm{T}$ current (IT) evoked at $-30 \mathrm{mV}$ as a function of the duration $(\Delta \mathrm{t})$ of the preceding hyperpolarization to $-100 \mathrm{mV}$ (see diagram of protocol). Note the biphasic shape of the curve with an increase in current amplitude due to a progressive recovery from inactivation of the channel population occurring during the first second combined with a slow decrease in current amplitude when the hyperpolarizing pre-pulse is lengthened. This decrease in amplitude mimics the progressive dephosphorylation of the native channels that takes place upon hyperpolarization. The maximal current is obtained with a 1-sec long hyperpolarization. B. Voltage dependency of the T-current potentiation. Superimposed traces of the simulated currents evoked at $-30 \mathrm{mV}$ after conditioning pre-pulse at various potential $(\Delta \mathrm{V})$. Each step depolarization to $-30 \mathrm{mV}$ was preceded by a 1 sec hyperpolarization to $-100 \mathrm{mV}$ to allow recovery from inactivation. Increasing the pre-pulse potential enhances the number of channels in mode 2 (fastest activation kinetics) leading to an increase in the T-current amplitude. Note the crisscrossing of the current waveforms, which indicates the acceleration in the inactivation kinetics that accompanies the current potentiation. The voltage-dependence of this increase in current amplitude was quantified in the graph below by plotting the ratio between the maximal current amplitude (IT $-30 \mathrm{mV})$ divided by the amplitude of the current conditioned by pre-pulse of varying potentials. Data were fitted by a modified Boltzman equation: a $/\left[1+\exp \left(-\left(\mathrm{V}-\mathrm{V}_{1} / 2\right) / \mathrm{k}\right)\right]+1$ with $\mathrm{V}_{1} / 2=-85.2 \mathrm{mV}, \mathrm{k}=4.1$ and a $=0.4 \mathrm{C}$. Kinetics of the T-current potentiation. Top: Simulated currents were evoked at $-30 \mathrm{mV}$ after a conditioning depolarizing pre-pulse to the same potential of increasing duration $(\Delta \mathrm{T}$; range, zero $\mathrm{msec}$ to $3 \mathrm{sec}$ ). A 1 -sec hyperpolarization to $-100 \mathrm{mV}$ was inserted between the conditioning pre-pulse and the step depolarization to remove channel inactivation (see protocol in insert). The graph presents the ratio between the amplitude obtained with the conditioning pre-pulse of a given duration $\Delta \mathrm{T}$ divided by the amplitude of the current obtained without pre-pulse $(\Delta \mathrm{T}=\mathrm{o} \mathrm{ms})$. Reflecting the increased amount of channels operating in mode 2 , the current is slowly potentiated by depolarizing pre-pulse of increasing duration, yielding a maximal current for depolarizations $>2$ sec. Data were fitted by a mono-exponential function with a time constant of $336 \mathrm{msec}$. Bottom: Starting from a holding potential of $-30 \mathrm{mV}$, where a maximal fraction of channels operates in mode 2 (see B), simulated currents were evoked at $-30 \mathrm{mV}$ after a hyperpolarizing pre-pulse to $-100 \mathrm{mV}$ of increasing duration $(\Delta \mathrm{t}$; range, $1 \mathrm{sec}$ to $20 \mathrm{sec}$; see protocol in insert) and compared successively to that obtained after the 20 sec hyperpolarizing pre-pulse. Note the progressive decrease in the current amplitude ratio indicating the progressive backward shift of the channel activity from mode 2 to mode 1 . Data were fitted by a mono-exponential function with a time constant of $2.7 \mathrm{sec}$. D. Steady state inactivation of simulated T-currents. The amplitude of the current evoked at -30 mV after conditioning prepulse of infinite duration to various potentials (from -130 to $-40 \mathrm{mV}$ ) was used to construct the classical steady state inactivation curve of the simulated current. Boltzman fit of the curve estimates a potential for half inactivation of $-80.5 \mathrm{mV}$. E. I-V curves of potentiated and non-potentiated currents. In both cases, I-V curves were constructed by successive step depolarizations of increasing potentials. To get the maximal channel population to operate in mode 1 , step depolarizations were performed from a holding potential of $-100 \mathrm{mV}$ of infinite duration (open circles). Conversely, a holding potential of $-30 \mathrm{mV}$ was used to obtain a maximal fraction of channels operating in mode 2 (filled circles). In the latter case, the step depolarizations were preceded by a $1-\mathrm{sec}$ long hyperpolarization to $-100 \mathrm{mV}$ to allow the channels to recover from inactivation. Boltzman fit of the I-V curves indicate a potential of half activation of $-51.1 \mathrm{mV}$ and $-54 \mathrm{mV}$ for nonpotentiated and potentiated currents, respectively. 
B
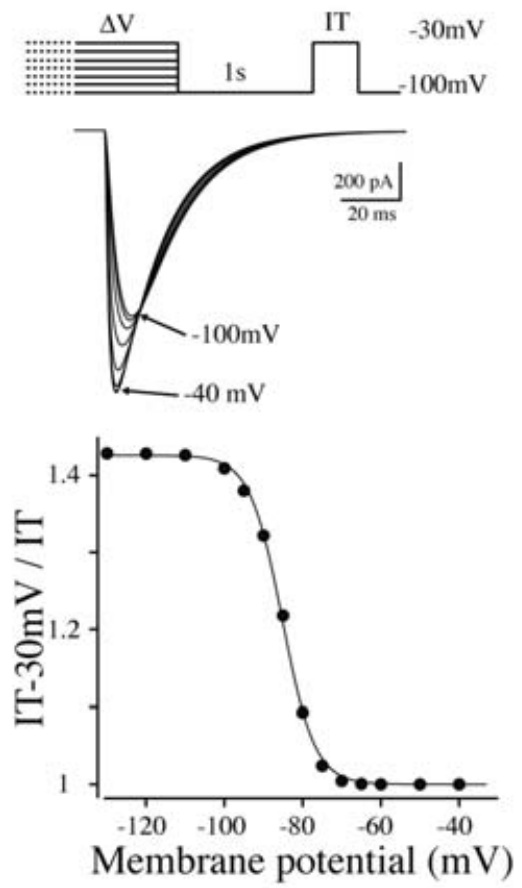

D

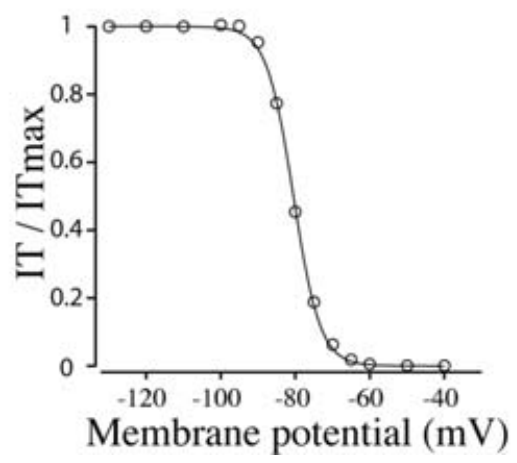

C
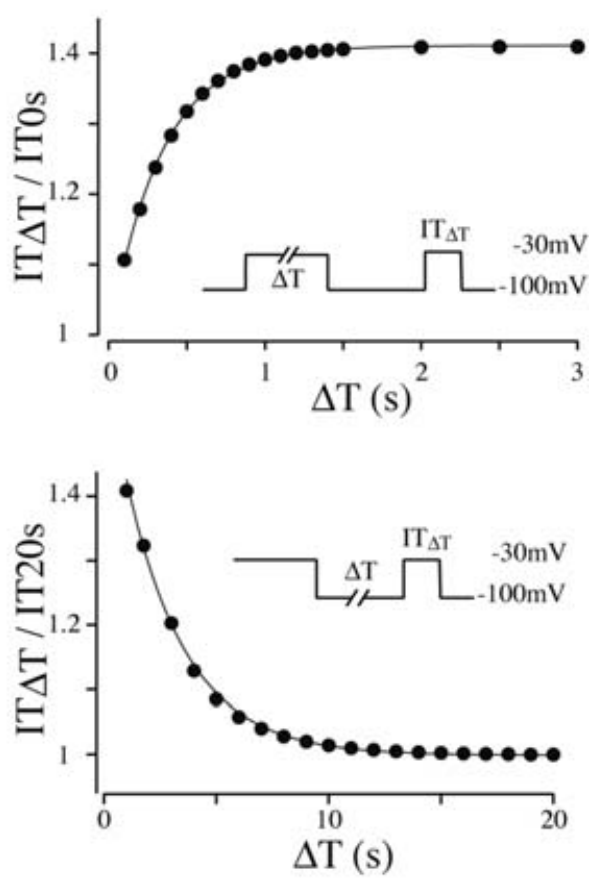

E

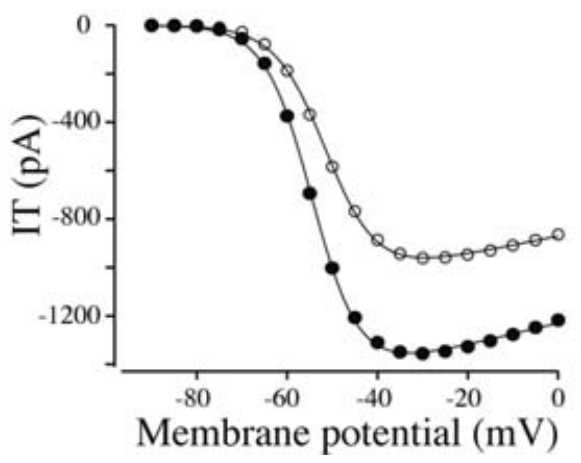

Fig. 3. Continued.

recorded thalamocortical neurons $(\sim 25 \%)$ and this does not correlate with the magnitude of the current potentation. In addition, the differences in rise time of the simulated current that need to be implemented in the model to induce the experimentally observed 1.5 -fold potentiation of the current amplitude were larger than those observed in situ. These discrepancies might be attributed to experimental limitations. Indeed, whole-cell somatic recordings of currents that originate partly from the dendrites are systematically associated to space-clamp errors (Destexhe et al., 1998) and to a filtering of the current that slows the fast onset kinetics of the currents and blurs their differences.

Regulation of the T-current does not occur in either dissociated neurons or nucleated excised patches (data not shown). Therefore, more accurate biophysical experimental data and confirmation of the hypothesis indicated by our modeling results needs recordings of unitary T-channel activities in the cell-attached mode. Although technically challenging, such recordings have been performed by Williams and Stuart (Williams and Stuart, 2000) in the soma and dendrites of the rat lateral geniculate thalamocortical neurons, where we described a clear ATP and voltage-dependent potentiation of the T-current (Leresche et al., 2004). However, the protocols used by Williams and Stuart (Williams and Stuart, 2000) do not isolate potentiated versus non-potentiated channels, so their results cannot be extrapolated to confirm our hypothesis.

Nevertheless, they do point to the existence of multiple T-channel populations in thalamocortical neurons with a non-uniform dendritic distribution and some specific biophysical properties. The existence of non-conventional T-currents was also indicated indirectly by current-clamp recordings in the cat lateral geniculate neurons that show the presence of high-threshold spikes with a pharmacological profile similar to that of T-channels (Hughes et al., 2004). Therefore, although in situ hybridization data have shown a clearly predominance of the Cav3.1 channel isotype in thalamocortical neurons (Talley et al., 1999), the potential that different channel subpopulations contribute to the macroscopic T-current cannot be eliminated fully at present. In this respect, it will be useful to determine whether the current generated by one of these subpopulations is closely 

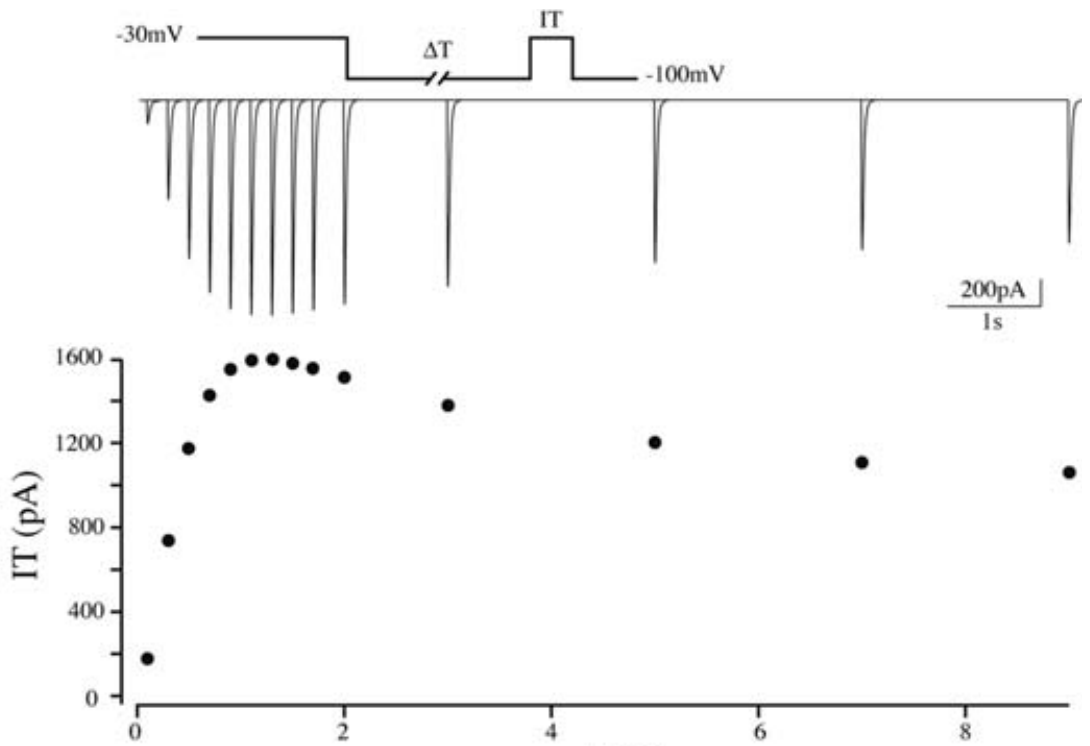

B
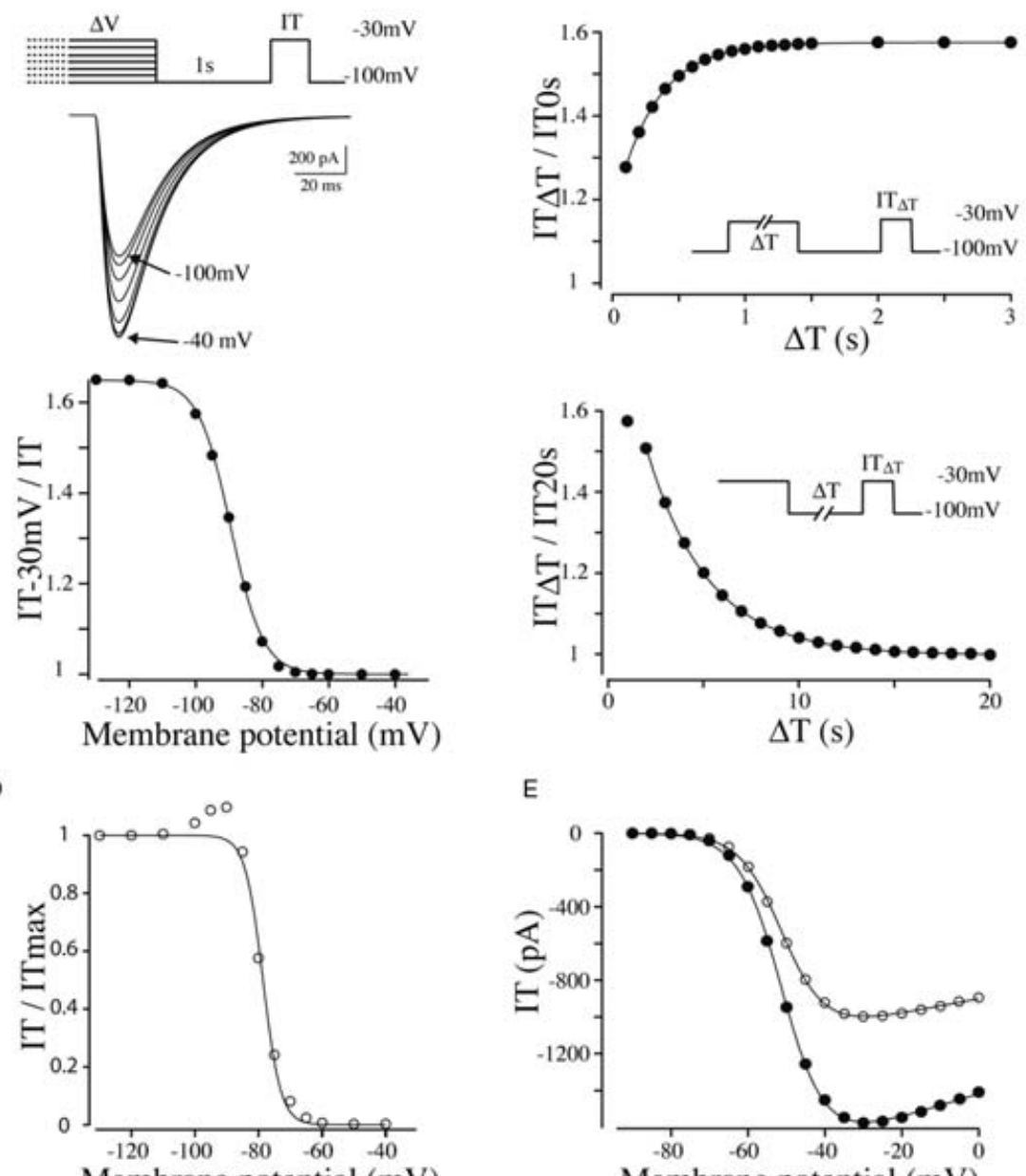

E

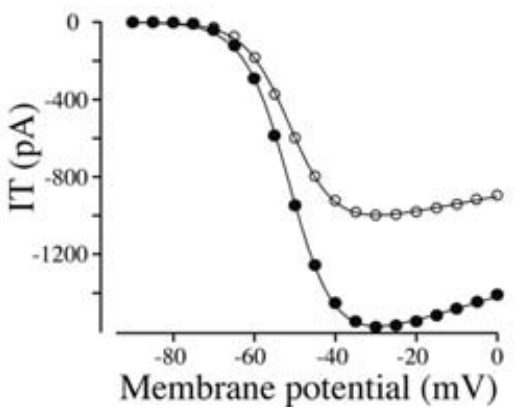

Fig. 4. Phosphorylation-induced recruitment of an additional population of channels mimics the major features of the T-current potentiation. A. Recovery from inactivation of the simulated current. (Protocol as in Fig. 3A.) B. Voltage dependency of the T-current potentiation. (Protocol as in Fig. 3B.) Data were fitted by a modified Boltzman equation: a $/\left[1+\exp \left(-\left(\mathrm{V}-\mathrm{V}_{1} / 2\right) / \mathrm{k}\right)\right]+1$ with $\mathrm{V}_{1} / 2=-89.5 \mathrm{mV}, \mathrm{k}=4.8$ and a $=0.6$. C. Kinetics of the T-current potentiation. (Protocol as in Fig. 3C.) Data were fitted by a mono-exponential function with time constants of 303 msec (on-time constant) and 3.2 sec (off-time constant). D. Steady-state inactivation of simulated T-currents. (Protocol as in Fig. $3 \mathrm{D}$.) Note the peculiar shape of the curve for conditioning pre-pulses around -100 mV (see Results for details). Boltzman fit of the curve estimates a potential for half inactivation of $-78.6 \mathrm{mV}$. E. I-V curves of potentiated and non-potentiated currents. (Protocol as in Fig. 3E.) Boltzman fit of the I-V curves indicate a potential of half activation of $-51 \mathrm{mV}$ for both non-potentiated and potentiated currents. 
dependent upon phosphorylation, as tested in our second series of simulations.

An additional hypothesis might also explain the increase in current amplitude induced by the channel phosphorylation. If $\mathrm{T}$-channels display multiconductance levels, one might imagine that the phosphorylation process might enhance the probability that the channel is in open states associated with the larger conductance levels. However, although the presence of multiconductance levels in native, low-threshold $\mathrm{Ca}^{2+}$ channels has been suggested, little is known at the singlechannel level for Cav3.1 channels (Reviewed in Talavera and Nilius, 2006).

To date, various models of thalamic T-current have been introduced for thalamocortical neurons, the majority of which are based on the Hodgkin-Huxley formalism (Wang et al., 1991; Huguenard and McCormick, 1992; McCormick and Huguenard, 1992; Toth and Crunelli, 1992; Williams et al., 1997; Destexhe et al., 1998). Initially, these models were incorporated in a single somatic compartment, and reproduced with accuracy the two intrinsic firing modes of thalamic neurons (i.e. bursting and tonic firing). The development of multicompartment models allows the use of various T-current densities in the somatic and dendritic compartments, together with other electrically active properties, and reproduces additional types of intrinsic oscillations as high-threshold oscillations (Rhodes and Llinas, 2005). Here, we had to use a model that allowed us to manipulate more finely the gating properties of the T-channel.

Different gating schemes with several similar features have been proposed for reconstituted or native channels in other tissue (Droogmans and Nilius, 1989; Chen and Hess, 1990; Serrano et al., 1999; Destexhe and Huguenard, 2000; Frazier et al., 2001; Burgess et al., 2002). We chose to adapt the scheme proposed by Burgess et al. (Burgess et al., 2002) and developed by Talavera et al. (Talavera et al., 2003; Talavera et al., 2004) to simulate the properties of Cav3.1 and Cav3.2 currents recorded in nearly physiological conditions. The latter uses the minimal number of conformational states necessary to reproduce the current properties and the gating charge movements that are associated with channel transitions (Burgess et al., 2002). Our gating-model that incorporates various states of the channels combined with an ATP-dependent regulatory mechanism, is more complex than the Hodgin-Huxley-like models that are generally used to simulate thalamocortical neuronal excitability. Although the use of our model increases computational time, its complexity introduces flexibility in the behavior of the T-type current that might help to unravel new roles in its ability to shape the different firing properties of thalamocortical neurons.

\section{ACKNDWLEDGEMENTS}

This work was supported by the ANR Neurosciences, Neurologie et Psychatrie 2006 project T-STATE.

\section{REFERENCES}

Burgess D.E., Crawford O., Delisle B.P. and Satin J. (2002) Mechanism of inactivation gating of human T-type (low-voltage activated) calcium channels. Biophysics Journal 82, 1894-1906.
Chen C.F. and Hess P. (1990) Mechanism of gating of T-type calcium channels. Journal of General Physiology 96, 603-630.

Coulter D.A., Huguenard J.R. and Prince D.A. (1989) Calcium currents in rat thalamocortical relay neurones: kinetic properties of the transient low-threshold current. Journal of Physiology (London) 414, 587604.

Crunelli V., Kelly J.S., Leresche N. and Pirchio M. (1987) The ventral and dorsal lateral geniculate nucleus of the rat: intracellular recordings in vitro. Journal of Physiology (London) 384, 587-601.

Crunelli V., Lightowler S. and Pollard C.E. (1989) A T-type Ca2+ current underlies low-threshold $\mathrm{Ca}+$ potentials in cells of the cat and rat lateral geniculate nucleus. Journal of Physiology (London) $413,543-561$.

Deschenes M., Paradis M., Roy J.P. and Steriade M. (1984) Electrophysiology of neurons of lateral thalamic nuclei in cat: resting properties and burst discharges. Journal of Neurophysiology 51, $1196-1219$.

Destexhe A. and Huguenard J.R. (2000) Which formalism to use for modeling voltage-dependent conductances? In Computational Neuroscience: Realistic Modeling for Experimentalists. pp. 129-157.

Destexhe A., Neubig M., Ulrich D. and Huguenard J. (1998) Dendritic low-threshold calcium currents in thalamic relay cells. Journal of Neuroscience 18, 3574-3588.

Droogmans G. and Nilius B. (1989) Kinetic properties of the cardiac T-type calcium channel in the guinea-pig. Journal of Physiology 419, $627-650$.

Frazier C.J., Serrano J.R., George E.G., Yu X., Viswanathan A., Perez-Reyes E. et al. (2001) Gating kinetics of the alpha1I T-type calcium channel. Journal of General Physiology 118, 457-470.

Hernandez-Cruz A. and Pape H.C. (1989) Identification of two calcium currents in acutely dissociated neurons from the rat lateral geniculate nucleus. Journal of Neurophysiology 61, 1270-1283.

Hines M.L. and Carnevale N.T. (1997) The NEURON simulation environment. Neural Computation 9, 1179-1209.

Hughes S.W., Lorincz M., Cope D.W., Blethyn K.L., Kekesi K.A., Parri H.R. et al. (2004) Synchronized oscillations at alpha and theta frequencies in the lateral geniculate nucleus. Neuron 42, 253268.

Huguenard J.R. and McCormick D.A. (1992) Simulation of the currents involved in rhythmic oscillations in thalamic relay neurons. Journal of Neurophysiology 68, 1373-1383.

Huguenard J.R. and Prince D.A. (1992) A novel T-type current underlies prolonged $\mathrm{Ca}(2+)$-dependent burst firing in GABAergic neurons of rat thalamic reticular nucleus. Journal of Neuroscience 12, 3804-3817.

Jahnsen H. and Llinas R. (1984) Electrophysiological properties of guinea-pig thalamic neurones: an in vitro study. Journal of Physiology 349, 205-226.

Lambert R.C., Bessaïh T. and Leresche N. (2006) Modulation of neuronal T-type calcium channels. CNS \& Neurological Disorders - Drug Targets 5 .

Leresche N., Hering J. and Lambert R.C. (2004) Paradoxical potentiation of neuronal T-type $\mathrm{Ca} 2+$ current by ATP at resting membrane potential. Journal of Neuroscience 24, 5592-5602.

Llinás R. and Jahnsen H. (1982) Electrophysiology of mammalian thalamic neurones in vitro. Nature 297, 406-408.

Llinás R., Ribary U., Jeanmonod D., Kronberg E. and Mitra P.P. (1999) Thalamocortical dysrhythmia: A neurological and neuropsychiatric syndrome characterized by magnetoencephalography. Proceedings of the National Academy of Science of the U.S.A. 96, 15222-15227. 
McCormick D.A. and Huguenard J.R. (1992) A model of the electrophysiological properties of thalamocortical relay neurons. Journal of Neurophysiology 68, 1384-1400.

Rhodes P.A. and Llinas R. (2005) A model of thalamocortical relay cells. Journal of Physiology 565, 765-781.

Serrano J.R., Perez-Reyes E. and Jones S.W. (1999) State-dependent inactivation of the alpha1G T-type calcium channel. Journal of General Physiology 114, 185-201.

Steriade M., McCormick D.A. and Sejnowski T.J. (1993) Thalamocortical oscillations in the sleeping and aroused brain. Science 262, 679-685.

Talavera K., Janssens A., Klugbauer N., Droogmans G. and Nilius B. (2003) Pore structure influences gating properties of the T-type Ca2+ channel alpha1G. Journal of General Physiology 121, 529-540.

Talavera K. and Nilius B. (2006) Biophysics and structure-function relationship of T-type Ca2+ channels. Cell Calcium 40, 97-114.

Talavera K., Staes M., Janssens A., Droogmans G. and Nilius B. (2004) Mechanism of arachidonic acid modulation of the T-type Ca2+ channel alpha1G. Journal of General Physiology 124, 225-238.

Talley E.M., Cribbs L.L., Lee J.H., Daud A., Perez-Reyes E. and Bayliss D.A. (1999) Differential distribution of three members of a gene family encoding low voltage-activated (T-type) calcium channels. Journal of Neuroscience 19, 1895-1911.

Toth T. and Crunelli V. (1992) Computer simulation of the pacemaker oscillations of thalamocortical cells. Neuroreport 3, 65-68.
Wang X.J., Rinzel J. and Rogawski M.A. (1991) A model of the T-type calcium current and the low-threshold spike in thalamic neurons. Journal of Neurophysiology 66, 839-850.

Williams S.R. and Stuart G.J. (2000) Action potential backpropagation and somato-dendritic distribution of ion channels in thalamocortical neurons. Journal of Neuroscience 20, 1307-1317.

Williams S.R., Toth T.I., Turner J.P., Hughes S.W. and Crunelli V. (1997) The 'window' component of the low threshold Ca2+ current produces input signal amplification and bistability in cat and rat thalamocortical neurones. Journal of Physiology (London) 505, 689-705.

Zhan X.J., Cox C.L., Rinzel J. and Sherman S.M. (1999) Current clamp and modeling studies of low-threshold calcium spikes in cells of the cat's lateral geniculate nucleus. Journal of Neurophysiology 81, $2360-2373$.

\section{Correspondence should be addressed to:}

Régis C. Lambert

Laboratoire de Neurobiologie des Processus Adaptatifs Université Pierre et Marie Curie-Paris6

Case 16

9 quai St Bernard 75005 Paris,

France

phone: + 33144272583

email: regis.lambert@snv.jussieu.fr 